\title{
Procedimentos Cirúrgicos Eleitos nas Condutas de uma Série de Casos de Glaucoma Congênito na Rede Pública de Campo Grande - MS
}

\section{Surgical Procedures Elected in the Conduct of a Series of Congenital Glaucoma Cases in the Public Health System of Campo Grande - MS}

\author{
Giovanna Danielson de Oliveira Silva ${ }^{\mathrm{a}}$; João Pedro de Novaes Corrêa ${ }^{\mathrm{a}}$; Julia Agnes Eneas de Almeida*a; \\ Juliana Hiroyo Moribe ${ }^{\text {a }}$, Karen de Souza Flores ${ }^{\text {a }}$ Tânia Gisela Biberg-Salum ${ }^{\text {ab }}$ \\ ${ }^{a}$ Universidade para o Desenvolvimento do Estado e da Região do Pantanal. MS, Brasil. \\ bUniversidade Estadual de Mato Grosso do Sul. MS, Brasil. \\ *E-mail: juuagnes@gmail.com
}

\begin{abstract}
Resumo
A cegueira é definida como a incapacidade de captação de informações do mundo através do sentido da visão. Em recém-nascidos e crianças, o glaucoma congênito é uma das principais patologias desencadeantes da cegueira, sendo o diagnóstico precoce fundamental para orientar o tratamento e alterar o prognóstico da patologia. A literatura, no geral, traz o tratamento cirúrgico como primeira linha adotada na abordagem do Glaucoma Congênito, sendo as principais técnicas utilizadas a goniotomia e a trabeculotomia. Tendo em vista a escassez de relatos na literatura local a esse respeito, o objetivo deste artigo consiste em verificar a conduta mais prevalente em uma série de casos de Glaucoma Congênito na Rede Pública do Mato Grosso do Sul. Este estudo é do tipo descritivo, quali-quantitativo, observacional, retrospectivo, baseado em análise dos registros nos prontuários dos pacientes atendidos na rede pública de saúde de Campo Grande - MS. Dos oito casos analisados, um sofreu evisceração com colocação de prótese, dois não tiveram o nome da técnica cirúrgica relatada e os outros cinco realizaram trabeculectomia. Apesar da maioria dos casos encontrados ter sido abordada com o tratamento cirúrgico, conforme o preconizado pela literatura, o procedimento eleito na maioria dos casos, a trabeculectomia, difere daquele citado na literatura como o de primeira escolha.
\end{abstract}

Palavras-chave: Glaucoma. Resultado de Tratamento. Trabeculectomia.

\begin{abstract}
Blindness is defined as the inability to capture information from the world through the sense of sight. In newborns and children, congenital glaucoma is one of the main causes of blindness, and early diagnosis is fundamental to guide treatment and change the prognosis of the condition. The literature adopts surgical treatment as the first line for the approach of Congenital Glaucoma, the main techniques being used for goniotomy and trabeculotomy. The objective of this article is to verify the most prevalent behavior in the series of cases of Congenital Glaucoma in the Public Network of MS, considering the scarcity in the literature, in order to evaluate if the techniques adopted in clinical practice follow what the medicine based in evidences presents as more appropriate. This is a descriptive, qualitative, observational, retrospective study, based on the records analysis in the medical patients records attended in the public health network in Campo Grande - MS. Of the eight analyzed cases, one had evisceration with prosthesis placement, two had no name of the surgical technique reported and the other five underwent trabeculectomy. Although most of the cases are treated with the surgical treatment as recommended by the literature, the procedure of first choice is the goniotomy technique. Differently from what was found in the ducts of the analyzed cases, the majority were treated with trabeculotomy. It is believed that the significant use of this technique relates to its greater ease of execution, transmitting more operational safety to the surgeons.
\end{abstract}

Keywords: Glaucoma. Treatment Outcome. Trabeculectomy.

\section{Introdução}

A cegueira é definida como a incapacidade de captação de informações do mundo através do sentido da visão (BRASIL, 2018), sendo essa uma condição que impacta as relações pessoais e sociais do indivíduo. Entre as suas etiologias, o glaucoma e suas derivações são considerados a primeira causa não curável (BRINK; BRASIL; BRINK, 2015). Em recém-nascidos e crianças, o glaucoma congênito é uma das principais patologias desencadeantes da cegueira, e consiste em uma má formação hereditária do mecanismo de drenagem do humor aquoso, associada ao aumento da pressão intraocular (FOSTER; GILBERT; JOHNSON, 2008). Sendo a principal manifestação clínica a tríade clássica: lacrimejamento excessivo crônico, fotofobia e blefaroespasmo (VIEIRA et al, 2018).
No que tange aos aspectos epidemiológicos, é importante salientar que esta é uma patologia rara que atinge cerca de 1 para cada 10.000 nascidos vivos e sua incidência pode ser variada em relação à raça e continente. No caso dos países Ocidentais, a prevalência apresentada pode chegar a 1 para cada 30.000 nascidos vivos (MANDAL; CHAKRABARTI, 2011). Além disso, há com maior frequência o acometimento do sexo masculino em relação ao sexo feminino, em aproximadamente $60 \%$ dos casos, com comprometimento bilateral em um quarto dos casos (NUNES; LOMÔNACO, 2008).

Apesar de se tratar de uma doença rara, é a causa mais comum de glaucoma na infância, sendo responsável por uma alta porcentagem de cegueira infantil não curável em todo o mundo (MOORE; TOMKINS; BEN-ZION, 2013), demonstrando-se assim a importância do diagnóstico precoce, fundamental para 
orientar o tratamento e alterar o prognóstico da patologia.

Atualmente, o tratamento é composto pelas vertentes medicamentosas e cirúrgicas. A literatura, no geral, adota o tratamento cirúrgico como primeira linha para a abordagem do Glaucoma Congênito, sendo as principais técnicas utilizadas a goniotomia e a trabeculotomia (PASSOS; KEIFER; AMADOR, 2009). Ambas consistem em seccionar o tecido mesodérmico, que impede a drenagem do humor aquoso. A goniotomia é indicada para córneas claras e proporciona lesões mínimas na conjuntiva e nos tecidos adjacentes, sendo a primeira opção sempre que possível. Por outro lado, nos casos em que essa não pode ser utilizada, a trabeculotomia passa ser a escolha subsequente, por ser uma técnica que pode ser realizada em córneas opacas (SILVA, 2016). Por fim, nos casos em que nenhuma das duas técnicas anteriores possa ser usada, a Trabeculectomia surge como uma alternativa de conduta, normalmente reservada como última opção em função do fato de causar maiores complicações que as anteriores (SOCIEDADE BRASILEIRA DE GLAUCOMA, 2017). Além disso, quando não identificado o canal de Schlemm durante o procedimento de trabeculotomia, a cirurgia pode ser convertida em trabeculectomia (SOCIEDADE BRASILEIRA DE GLAUCOMA, 2017).

Sendo assim, o objetivo deste estudo consistiu em verificar a conduta cirúrgica mais prevalente em uma série de casos de Glaucoma Congênito na Rede Pública do Mato Grosso do Sul.

\section{Material e Métodos}

Este estudo é do tipo descritivo, quali-quantitativo, observacional, retrospectivo, baseado em análise dos registros nos prontuários dos pacientes atendidos nos serviços de oftalmologia da rede pública de saúde do município de Campo Grande - MS. A coleta de dados foi iniciada no dia 28 de junho de 2018, sendo finalizada no dia 25 de setembro do mesmo ano.

Foram incluídos, para a coleta de informações, os prontuários físicos e/ou digitais registrados com diagnóstico de glaucoma congênito e/ou de glaucoma em geral, identificados pelo CID H.40 (Glaucoma) ou pelo CID Q.15 (Glaucoma Congênito), cadastrados no período de janeiro de 2015 a setembro de 2018, cujo paciente apresentasse idade atual entre zero e 35 anos. Depois da triagem inicial de todos esses prontuários, foram selecionados apenas aqueles que apresentavam a etiologia congênita da doença, sendo excluídos os prontuários que não contivessem a idade atual do paciente. Foram apontados os dados acerca da idade, idade do diagnóstico, sexo, raça, olho acometido, quadro clínico, conduta terapêutica adotada e evolução do paciente. Os prontuários incompletos também foram incluídos na pesquisa por se tratar de uma doença rara, dessa forma, todos os pacientes com diagnóstico de GC no período proposto foram analisados.

Foram analisados prontuários armazenados fisicamente nos setores de oftalmologia dos seguintes locais: (1) Hospital São Julião, (2) Associação Beneficente de Campo
Grande/ Hospital Santa Casa, (3) Hospital Universitário Maria Aparecida Pedrossian e, digitalmente no (4) Centro de Especialidades Médicas e (5) Centro de Especialidades Infantis de Campo Grande - MS. A coleta ocorreu após prévia entrevista nas instituições para avaliação e confirmação da autorização do trabalho, bem como dos documentos apresentados. Este estudo foi aprovado junto ao Comitê de Ética em Pesquisa da Universidade Anhanguera UNIDERP, sob o número de parecer 2.542.279.

Os dados dos pacientes um e dois foram coletados na Associação Beneficente de Campo Grande/ Hospital Santa Casa, dos pacientes três, quatro, cinco e seis são referentes aos atendimentos do Hospital Universitário Maria Aparecida Pedrossian, dos pacientes sete e oito no Hospital São Julião, e do paciente nove no Centro de Especialidades Infantis de Campo Grande - MS. Com essas informações foi elaborado um quadro comparativo, permitindo uma visão abrangente do perfil de cada paciente, podendo com isso estes dados serem melhores analisados de forma a responder os objetivos da pesquisa.

\section{Resultados e Discussão}

Foram analisados prontuários de nove pacientes, sendo que seis $(66,67 \%)$ deles eram do sexo masculino e três $(33,33 \%)$ do sexo feminino. Em estudo realizado (BRINK; BRASIL; BRINK, 2015), encontra-se uma divergência em relação a essa proporção de gêneros, sendo que em um total de 32 pacientes com glaucoma congênito, 19 eram do gênero feminino $(59,37)$ e 13 eram do gênero masculino $(40,62)$. A acuidade visual alterada foi o achado mais presente $(55,56 \%)$.

Em relação à lateralidade do acometimento, ambos os olhos $(66,67 \%)$ foi o mais prevalente (Quadro 1). Entre os nove prontuários, os casos 2, 4, 5, 6, 7 e 8 apresentaram acometimento bilateral dos olhos, já os casos 1 e 3 tiveram acometimento unilateral (do olho esquerdo e do olho direito, respectivamente) e o caso 9 não foi esclarecido. Dados condizentes com o estudo analisado (BRINK; BRASIL; BRINK, 2015), que aponta a bilateralidade, como sendo mais prevalente, sendo encontrada em $91 \%$ dos pacientes.

Quadro 1 - Conduta e Desfecho dos pacientes analisados

\begin{tabular}{|c|c|c|c|}
\hline Paciente & Conduta & $\begin{array}{c}\text { Desfecho } \\
\text { quanto a PIO }\end{array}$ & $\begin{array}{c}\text { Desfecho } \\
\text { Acuidade }\end{array}$ \\
\hline 1 & TREC OE & PIO normal & SI \\
\hline 2 & TREC Bilateral & PIO aumentada & SI \\
\hline 3 & Evisceração & SI & Cegueira \\
\hline 4 & TREC Bilateral & PIO normal & SI \\
\hline 5 & TCNE & PIO aumentada & SI \\
\hline 6 & TCNE & PIO normal & SI \\
\hline 7 & TREC & SI & Visão subnormal \\
\hline 8 & TREC & SI & Cegueira \\
\hline 9 & SI & SI & SI \\
\hline
\end{tabular}

Legenda: PIO (Pressão Intraocular), SI (Sem Informações no Prontuário), TCNE (Técnica Cirúrgica Não Especificada) e TREC (Trabeculectomia).

Fonte: Dados da pesquisa. 
Em se tratando da conduta adotada para os casos em questão, dos nove pacientes citados, cinco foram tratados com a associação dos métodos, cirúrgico e medicamentoso $(55,56 \%)$, três foram tratados exclusivamente com o método cirúrgico $(33,33 \%)$ e, em um deles não foi descrita a conduta terapêutica em seu prontuário (11,11\%) (Quadro 1).

Dos oito pacientes analisados com dados no prontuário, um sofreu evisceração do olho direito com colocação de prótese $(11,11 \%)$, dois não tiveram o nome da técnica cirúrgica relatada $(22,22 \%)$ e os outros cinco $(55,56 \%)$ realizaram trabeculectomia (TREC) (Quadro 1).

Como pode se depreender do citado acima, a maioria dos casos encontrados foi abordada com o tratamento cirúrgico conforme o preconizado pela literatura, ainda que a opção dos cirurgiões tenha sido pela trabeculectomia, a despeito da goniotomia ser a técnica recomendada como primeira escolha e, quando esta não é possível, em função da opacidades corneanas, a trabeculotomia ser o procedimento indicado (PASSOS; KEIFER; AMADOR, 2009; BRINK; BRASIL; BRINK, 2015). A trabeculectomia conforme o demonstrado pela literatura seria a última técnica alternativa, pois apresenta maior potencial de complicações pós-operatórias causadas, principalmente, pela fibrose excessiva em pacientes jovens, apesar de os estudos apontarem para taxas de sucesso semelhantes entre as diferentes técnicas utilizadas (MANDAL; CHAKRABARTI, 2011).

Sendo assim, muitos médicos optam pela trabeculectomia em função da menor complexidade no procedimento cirúrgico ou por estarem mais familiarizados e habilitados com esta técnica, em se comparando com o manejo das peculiaridades das anomalias anatômicas que, frequentemente, são encontradas nos casos de glaucoma congênito (SOCIEDADE BRASILEIRA DE GLAUCOMA, 2017), gerando mais segurança operacional aos médicos que as realizaram. Além disso, estudos comprovam que a TREC tem se mostrado eficaz na diminuição da pressão intraocular e no controle da progressão da doença (CRONEMBERGER et al., 2004).

Quanto à abordagem do momento de realização da intervenção cirúrgica se encontrou que a idade dos pacientes, de forma geral, variou entre 1 e 32 anos, sendo que a idade de realização da cirurgia, em um deles $(11,11 \%)$ foi entre 3 meses e 1 ano, em 5 deles $(55,55 \%)$ foi com mais de um ano, e em dois $(22,22 \%)$ dos pacientes esses dados não constavam no prontuário. Sabe-se que a idade de realização da cirurgia é de grande importância prognóstica, uma vez que crianças entre três meses e um ano de vida apresentam melhor prognóstico chegando a $90 \%$ de sucesso quando da realização da goniotomia ou da trabeculotomia. Em contrapartida, em recém-nascidos e em maiores de um ano, essa taxa é reduzida a $30 \%$ de sucesso (SOCIEDADE BRASILEIRA DE GLAUCOMA, 2017). Por outro lado, em relação à técnica de trabeculectomia, na busca por referências não foram encontrados dados que correlacionam a idade da intervenção com o prognóstico do paciente.
Ao analisar trabalhos presentes na literatura, existem diversas pesquisas comparando os índices de sucesso entre a goniotomia e a trabeculotomia, que variam em sua porcentagem de sucesso. Um estudo realizado com 35 olhos, de 23 pacientes portadores de GC primário submetidas à trabeculotomia, 88,5\% foram operados antes de 1 ano de idade e o índice de sucesso absoluto foi de $62,9 \%$ e relativo de $22,8 \%$, com sucesso total de $85,7 \%$ demonstrando o sucesso da técnica (MEIRELLES et al., 2004).

Um estudo, que avaliou 820 olhos de 532 pacientes com diagnóstico de GC até 1 ano de idade, submetidos a goniotomia, trabeculotomia ou trabeculotomiatrabeculectomia combinada, relatou sucesso de 81 a $100 \%$ para a forma leve de GC; sucesso de 13\%, 40\% e $80 \%$ na forma moderada, respectivamente, para goniotomia, trabeculotomia e trabeculotomia-trabeculectomia e $10 \%$ e $70 \%$ para grave por trabeculotomia e cirurgia combinada, respectivamente. Tal estudo correlacionou a classificação clínica do GC com a decisão cirúrgica, uma vez que a forma leve apresentou alto sucesso cirúrgico, independentemente do procedimento escolhido, apesar de, na forma moderada, a trabeculotomiatrabeculectomia combinada ter mostrado melhores resultados (AL-HAZMI et al., 2004).

Outra pesquisa avaliou 165 olhos, de 112 crianças, dos quais $86,7 \%$ passaram por procedimentos combinados na proposta de cirurgia de primeira escolha. A taxa de reoperação apresentada nesta série de casos foi de $20 \%$, com média de 1,3 procedimentos por olho, sendo muito menor que as taxas de reoperação após trabeculotomia e goniotomia. Portanto, infere-se que o uso das técnicas combinadas, em pacientes com GC primário, é apontado como primeira escolha de tratamento, especialmente em casos graves com córnea nebulosa, visto a vantagem de que, na falha da trabeculotomia, pode-se convertê-la a TREC (BAYOUMI, 2016).

A presente casuística observou que eentre os casos com conduta cirúrgica, três $(33,33 \%)$ apresentaram desfechos favoráveis: paciente 1, 4 e 6 evoluíram com pressão intraocular (PIO) normal, apesar de não haver informação em relação à acuidade visual nos prontuários; cinco casos (55,55\%) obtiveram evolução desfavorável: pacientes 2 e 5 com PIO aumentada, pacientes 3 e 8 progrediram para cegueira e paciente 7 desenvolveu visão subnormal. $O$ paciente 9 apresentou dados inconclusivos em função da falta de informações sobre a evolução pós-cirúrgica deste paciente (Quadro 1).

A Organização Mundial de Saúde (OMS) define a "cegueira legal", na $10^{a}$ edição da Classificação Internacional de Doenças (CID-10), como sendo a acuidade visual menor que $20 / 400$ ou $3 / 60$ ou campo visual menor que vinte graus no olho menos prejudicado. Neste trabalho não foram obtidos os valores da acuidade visual dos pacientes 3 e 8 , uma vez que nos prontuários vinha relatada a cegueira como desfecho único apontado (SOCIEDADE BRASILEIRA DE VISÃO SUBNORMAL, 2019). 


\section{Conclusão}

No presente estudo se observa que a maioria dos pacientes afetados foi do sexo masculino, com acometimento de ambos os olhos, sendo os principais aspectos clínicos verificados na época do diagnóstico, a alteração da acuidade visual, buftalmo, megalocórnea e presença de leucoma.

Além disso, assim como observado na literatura, este estudo obteve resultados que demonstram a preferência de escolha pelo tratamento cirúrgico dos pacientes. No entanto, contrapondo-se com a sequência de escolha das condutas esperadas, conforme preconizado pela literatura vigente, a trabeculectomia se mostrou como sendo a técnica mais utilizada na rede pública de Campo Grande, MS.

\section{Referências}

AL-HAZMI, A. et al. Correlation between surgical success rate and severity of congenital glaucoma. Br. J. Ophthalmol., v.89, p.449-453, 2004. doi:10.1136/bjo.2004.047761

BAYOUMI, N.H.L. Surgical management of primary congenital glaucoma in egypt. J. Egypt. Ophthal. Soc., v.109, n.2, p. 85-92, 2016. doi:10.4103/2090-0686.193398

BRASIL. Protocolo Clínico e Diretrizes Terapêuticas Glaucoma. Brasília: MS, 2018.

BRINK, D.B.C.; BRASIL, M.B.Q.; BRINK, G.B. Perfil epidemiológico dos pacientes com glaucoma congênito atendidos no Hospital Regional de São José. Rev. Bras. Oftalm., v.74, n.3, p.133-137, 2015. doi:10.5935/0034-7280.20150029

CRONEMBERGER, S. et al. Ressecção de bolsa hiperfuncionante para tratamento de hipotonia ocular crônica - Relato de casos. Arq. Bras. Oftalm., v.67, n.4, p.637-44, 2004. doi:10.1590/ S0004-27492004000400014
FOSTER, A.; GILBERT, C.; JOHNSON, G. Changing patterns in global blindness: 1988-2008. Comm. Eye Health, v.21, n.67, p.37-39, 2008.

MANDAL, A.K.; CHAKRABARTI, D. Update on congenital glaucoma. Indian J. Ophthalm., v.59, n.59, p.148-157, 2011. doi:10.4103/0301-4738.73683

MEIRELLES, S.H.S. et al. Resultados em longo prazo da trabeculotomia no tratamento do glaucoma congênito primário. Rev. Bras. Oftalm., v.63, n.5, p.326-333, 2004.

MOORE, D.B.; TOMKINS, O.; BEN-ZION, I. A review of primary congenital glaucoma in the developing world. Surv. Ophthalmol., v.58, n.3, p.278-285, 2013. doi: 10.1016/j. survophthal.2012.11.003

NUNES, S.S.; LOMÔNACO, J.F.B. Desenvolvimento de conceitos em cegos congênitos: caminhos de aquisição do conhecimento. Psic. Escol. Educ., v.12, n.1, p.119-138, 2008. doi:10.1590/S1413-85572008000100009

PASSOS, A.F.; KEIFER, K.; AMADOR, R.C. Resultados da associação trabeculotomia-trabeculectomia em pacientes não idosos. Rev. Bras. Oftalm., v.68, n.4, p.223-230, 2009. doi:10.1590/S0034-72802009000400007

SILVA, I.M. Diagnóstico do glaucoma congênito: revisão sistemática. Salvador: Universidade Federal da Bahia, 2016.

SOCIEDADE BRASILEIRA DE GLAUCOMA. Consenso de Cirurgia de Glaucoma. São Paulo: Ipsis Gráfica e Editora, 2017.

SOCIEDADE BRASILEIRA DE VISÃO SUBNORMAL. Conselho Brasileiro de Oftalmologia Parecer técnico: Visão Monocular. São Paulo: Conselho Brasileiro de Oftalmologia, 2019.

VIEIRA, J.M. et al. Glaucoma congênito: desafios do diagnóstico precoce, tratamento e acompanhamento. Rev. Méd. Minas Gerais, v.28, n.7, p.6-9, 2018. doi: 10.5935/2238-3182.20180149. 